\title{
LA PROBLEMÁTICA ACERCA DE LAS DESIGUALDADES REGIONALES*
}

\author{
José LuIS CORAGgio \\ El Colegio de México
}

\section{INTRODUCCIÓN}

Usualmente, la cuestión regional es reducida a la existencia de desigualdades regionales en el seno de un país y eventualmente a la existencia de procesos acumulativos que tienden a reproducir o aún acentuar dichas desigualdades. Es también común la proposición de que mientras las desigualdades regionales están en disminución en los países desarrollados, en los países periféricos del sistema capitalista la tendencia sigue siendo a la creciente desigualdad, lo que se atribuye al débil desarrollo capitalista o a la posición subordinada que ocupan en el sistema mundial. Esta forma de formular la problemática está impregnada de los términos en que la misma se desarrolló en los centros académicos y de planificación de los países centrales. Si bien se encuentran numerosos intentos de superar estas formulaciones, dicha superación difícilmente podrá lograrse con base en una adaptación de aquellas teorías y métodos de análisis a las particulares condiciones de nuestros países. Entre otras cosas, esta dificultad se debe a que las mencionadas teorías no son tampoco aptas para dar cuenta de la cuestión regional en los países centrales. En suma, es necesario efectuar una ruptura teórica con la problemática específica dominante, como parte del proceso de formulación de la cuestión regional en América Latina. En tal sentido, intentaremos presentar nuestra visión de los términos usuales de dicha problemática como paso previo a esbozar algunas características que a nuestro juicio debería cumplir una formulación crítica de la cuestión regional en América Latina.

\footnotetext{
* Este artículo es una versión parcialmente modificada de la ponencia presentada al Seminario sobre desigualdades regionales organizado por el grupo de trabajo del mismo nombre (CLACSO), en Colonia Tovar, Venezuela (junio, 1977), bajo el título: "Algunas cuestiones relacionadas con el estudio de las desigualdades regionales en América Latina".
} 


\section{LA PROBLEMÁTICA DE LAS DESIGUALDADES REGIONALES EN SU VERSIÓN DOMINANTE}

Al intentar bosquejar una presentación de los términos usuales de la problemática acerca de las desigualdades regionales ${ }^{\mathcal{y}}$ partimos de la práctica misma de los especialistas en el tema, con la hipótesis de que encontraremos una unidad funcional entre cuatro elementos interdependientes de la misma: a) la manera en que se presentan las mencionadas desigualdades (descripción); $b$ ) los juicios de valor que sobre las situaciones descritas se realizan; c) los intentos de explicación de dichas desigualdades; d) las propuestas para modificar la situación al respecto (políticas). Al hacerlo no estamos siguiendo a ningún autor en particular, ni afirmamos que todo exponente de esta versión de la problemática recorre sistemáticamente los cuatro aspectos (unos pueden centrarse en intentos de una descripción más sofisticada, otros se limitan a calificar con abundancia de adjetivos una situación apenas analizada, otros se concentran en proponer medidas que supuestamente tenderían a una mayor igualdad interregional de los indicadores a partir de cualquier situación de partida, etc.). En realidad lo que haremos es "reconstruir" lo más coherentemente posible los términos de la problemática de las desigualdades regionales en su versión dominante, a fin de posteriormente proceder a su crítica.

\section{La descripción del fenómeno}

El procedimiento usualmente seguido puedg desglosarse en tres decisiones principales: a) adoptar una participación del territorio nacional en un conjunto de regiones no yuxtapuestas; $b$ ) seleccionar un vector de variables cuyo valor será medido o estimado para cada región; c) seleccionar uno o más indicadores sintéticos de la desigualdad interregional para cada variable o conjunto de variables.

Un manipulador avezado de estos métodos, sabe que existe una "interacción" entre las decisiones $a$ y $b$, en tanto con distintas regionalizaciones pueden obtenerse caracterizaciones muy diversas del grado de desigualdad interregional para una variable, y que una regionalización adecuada para describir la desigual distribución espacial de una variable puede ser inapropiada para otra. Sin embargo, en tanto las regiones son prácticamente consideradas como los "sujetos" entre los cuales debe constatarse una desigualdad, lo usual es sacrificar rigor en el análisis de la distribución espacial de cada variable para obtener una caracterización de la posición relativa de cada ente-región para las distintas variables. La cuestión de cómo se delimitan los "sujetos" o elementos del "sistema espacial", como a veces se lo denomina, suele estar contestada "exógenamente", al adoptar alguna regionalización ya existente.

\footnotetext{
1 En el texto utilizaremos indistintamente el término regionales o interregionales, aunque entendemos que el segundo se ajusta mejor al tema.
} 
La selección de las variables cuya distribución se estudiará suele tener un condicionante histórico aún más claro que en el caso de la regionalización. Normalmente se utiliza información secundaria, con lo que el espectro de posibles variables queda predeterminado por el sistema oficial contemporáneo de información estadística y sus apéndices privados. Los "datos disponibles" hacen las veces de "la realidad", y la determinación de las variables relevantes se convierte en una selección entre los mismos. Un método posible ante esta encrucijada es sencillamente no elegir, es decir, utilizar todas las variables disponibles, para tener un "panorama amplio" del espectro de desigualdades interregionales. Si este procedimiento se vuelve engorroso por la dificultad para interpretar tal masa de información, se suele utilizar últimamente el método de análisis de factores, a fin de condensar la información (con una pérdida mínima de "variación") en uno, dos o tres factores compuestos, normalmente interpretados con alguna denominación genérica, como "nivel de desarrollo", "estatus socioeconómico", etc. En cualquier caso, cada variable o factor adopta un valor numérico para cada elemento-región, constituyéndose en una caracterización media parcial de la misma (y por tanto implicándose una homogeneidad intrarregional, o al menos una heterogeneidad no relevante). Sería gratuito reproducir aquí la lista más o menos conocida de las variables que suelen utilizarse (o, si se quiere, de las que suelen tenerse datos). Sin duda la más mencionada es el ingreso (a veces con el aditamento: "real"), pero también sin duda la menos medida efectivamente a niyel regional. ${ }^{2}$ El problema del diferente tamaño de las regiones utilizadas lleva en muchos casos a utilizar alguna magnitud base como elemento ponderador para facilitar la comparación (usualmente la población de cada región). Un supuesto para la interpretación posterior en este tipo de análisis es que cada variable toma valores a lo largo de un continuo unidireccional donde una situación siempre puede compararse a otra como "mejor" o "peor". Así, por ejemplo, un mayor porcentaje de ocupación en el sector industrial será siempre mejor que uno menor, etc. Así, se ubica en un ordenamiento pretendidamente inequívico las regiones respecto a cada variable y, si hay una correlación satisfactoria, se llega a ubicarlas en un ordenamiento general para el conjunto de variables, utilizando diversas artimañas cuantitativas.

Debe destacarse que esta "operación descripción" no es en rigor independiente y efectivamente previa a los juicios de valor que provoca la situación regional, a la concepción del tipo de explicación que espera proveerse, o al tipo de posibles acciones que se tiene en mente. Por ejemplo, si se está pensando en términos de una explicación según los cánones de la estadística inferencial, sería un mal paso utilizar todas las va-

2 Ver, por ejemplo: D. Slater: "Regional Inequality in a Dependent SocioEconomic System - The Peruvian Case, 1945-1966", Progress in Planning, Vol. 4, pt. 2, 1975, quien tiene que utilizar, en "representación" de la variable ingreso real la de "número de vehículos motorizados de propiedad privada". 
riables disponibles para la descripción del fenómeno. Por el contrario, debería establecerse una distinción entre las variables que representan el fenómeno (dependientes) y las que hipotéticamente lo explicarían (independientes). Así, pueden colocarse en el primer grupo variables asociadas a algo así como el bienestar del habitante medio, y en el segundo otras asociadas a, por ejemplo, características de la estructura de producción. Lo que nos lleva a que, salvo en casos del empirismo más rampante (en rigor deberíamos llamarlo "datismo"), la determinación de las variables adecuadas para la descripción del fenómeno debería estar orientada desde cierta teoría o al menos visión acerca del fenómeno y de sus causas. En lo que hace a los juicios de valor anticipados, si tienen más que ver con consideraciones de equidad social o, en cambio, con cuestiones de eficiencia económica, obviamente ésto orienta también la selección de los indicadores. Por último, si, por ejemplo, se está pensando en promover una diferente distribución de las actividades industriales de modo de lograr una "igualación interregional de oportunidades" en cuanto a la probabilidad de que un habitante medio de una región tenga un empleo industrial, no tendría sentido utilizar como indicador de la desigualdad el número de empleos industriales por kilómetro cuadrado, dado que la distribución de la población misma no es uniforme.

En versiones relativamente sofisticadas, la aserción final de que existen (o no) desigualdades interregionales significativas, estaría fundamentada en el grado de significación obtenido según los "test" estadísticos de uso corriente. $\mathrm{Si}$ interesa individualizar las "regiones-problema", se utilizarán métodos que permitan además mantener la individualidad de cada región vis a vis el resto (utilizando, por ejemplo, los valores estandarizados para cada región respecto a cada variable o los valores factoriales (factor scores) para cada factor componente).$^{3}$

\section{Los juicios de valor}

Podríamos esquemáticamente afirmar que los juicios que suelen hacerse sobre las desigualdades interregionales constatadas están inspirados en uno de dos (o en ambos) criterios generales: el de equidad social y el de eficiencia económica en términos de crecimiento. Según predomine uno u otro aspecto, cierta desigualdad interregional específica será o no un "problema". Esto tiene además efectos sobre la determinación de los "patrones de referencia", es decir las distribuciones ideales que se adoptan como norma para cada variable y con la cual se compara la efectivamente existente a fin de constatar si la desviación de la norma es aceptable o no. Cuando predomina el criterio de equidad social, la norma que suele utilizarse es precisamente la de igualdad (equidistribución) entre regiones, con las debidas ponderaciones. Así, una situación hipotética de

3 Ver, por ejemplo: Slater, D., op. cit. 
igual producto bruto per capita nos permite clasificar a las regiones en las que están por debajo y las que sobrepasan la norma.

En cambio, cuando predomina el criterio de eficiencia económica difícilmente las normas propuestas serán las de una equidistribución. Aquí se hablará de grados de desigualdad que pueden alejarse del óptimo por excesiva desigualdad o por excesiva igualdad. Un caso claro es el de la conocida norma provista por la denominada "regla de rango-tamaño" para la distribución de la población urbana, que si bien no está pensado para regiones en el sentido que aquí se les da, tiene consecuencias sobre la variación de dicha población entre regiones. Así, una desigualdad regional estaría aquí dada por una excesiva concentración más que por el alejamiento de la equidistribución per se. Es evidente que estamos forzando un tanto el uso del término "igualdad" (lo que podría superficialmente resolverse reemplazándolo por los consabidos "armonía", "equilibrio", etc.), pero otro tanto podría decirse de la pauta obtenida por la distribución de una variable siguiendo la (desigual) distribución de una magnitud base. Por otra parte, formularlo así lleva a no cargar innecesariamente las tintas en la dicotomía "eficientistas versus igualitaristas", como si los primeros desecharan toda consideración congruente con la equidad. ${ }^{*}$

En otros términos, el eficientista discriminaría entre desigualdades inevitables (o "necesarias") y desigualdades corregibles (o innecesarias) e incluso nocivas desde el criterio que adopta. ${ }^{5}$

Mientras el predominio del criterio de equidad orientaría a una descripción con base en indicadores relacionados con "el bienestar" de la población, el predominio del criterio de eficiencia orientaría más bien hacia indicadores relacionados con desfasajes en la combinación de los recursos (grado de explotación de recursos naturales escasos, o grado de utilización de la fuerza de trabajo o del aparato productivo existente, costos de abastecimiento de bienes y servicios, variaciones en la productividad, etc., etc.). Así como el criterio predominante interactúa con la elaboración de la descripción, también tiene claras relaciones con el momento de las propuestas. El criterio de equidad se basa en la equidistribución como norma y las propuestas en general serán de redistribución para paliar la "injusticia interregional", pretendiendo los más de los casos actuar directamente sobre los efectos (si hay baja tasa de escolaridad, construir escuelas, etc.). El criterio de eficiencia se basa en pautas de referencia construidas a partir de modelos de asignación óptima de re-

4 Por ejemplo, un igualitarista a ultranza propondría: "todo niño en edad escolar debe estar dentro del radio de acceso a una escuela", mientras el eficientista diría "todo niño en edad escolar, ubicado en un área de tal densidad, debe estar dentro del radio de acceso a una escuela" (es decir, si es económicamente "viable").

5 Tal vez a esta altura sea importante reiterar que en esta primera parte nos esta mos moviendo (algo incómodamente) dentro de la problemática dominante, sin cues-
tionar aún sus términos. 
cursos, ${ }^{6}$ congruentes con propuestas de "racionalización" en las distribuciones espaciales.

Esta diferenciación entre criterios reguladores se hace menos nítida en algunos casos. Por ejemplo, cuando se utiliza la variable "tasa de crecimiento del producto regional" y se propone como norma su igualación interregional. Sin embargo, aún en este caso puede afirmarse que predomina el criterio de equidad, pues esta norma será vista por el "eficientista" como una restricción innecesaria al ritmo de crecimiento.

\section{La explicación del fenómeno y las propuestas para el cambio}

Constatada la existencia de desigualdades significativas tanto en lo que hace a las "condiciones medias de vida de la población" de distintas regiones, como a los ritmos y pautas de crecimiento económico, y enjuiciadas las mismas como "injustas", "ineficientes", etc., vendría el momento del "¿qué hacer?". Una alternativa es pasar, sin mediaciones, de la descripción del fenómeno y de su comparación con normas o "imágenes objetivo", a armar propuestas de acción directa para modificar la desigualdad en los indicadores según las prioridades que de algún modo se establezcan. Sin embargo, fácilmente se llega a la conclusión de que entre los diversos aspectos del fenómeno de desigualdades interregionales existe una interdependencia muy marcada, con lo que no puede adoptarse un supuesto de aditividad e independencia de las diversas acciones propuestas. En tal sentido, se hace necesaria una jerarquización de dichos aspectos, en términos de proceso o al menos de su versión más simplista: la de mecanismo. Se requiere entonces una concepción teórica del proceso o mecanismo -que genera esos efectos de desigualdad o de qué manera interdependiente las desigualdades se alimentan unas a otras.

Tal como mencionáremos más arriba, una alternativa sería sencillamente postular que existe una dependencia unilateral de ciertas variables respecto a otras (en el sentido que la evolución de los valores de las segundas determinan los de las primeras). Si esta hipótesis puede sustentarse estadísticamente al comprobar una asociación significativa entre las variables de uno y otro grupo, tendríamos una "explicación" que a su vez nos indicaría con qué intensidad debemos actuar sobre las variables independientes para modificar las dependientes en cierto grado. Este tipo de orientación está presente cuando, por ejemplo, se critica al "asistencialismo"

- Cabe señalar que, en rigor, dichos modelos suelen servir exclusivamente como "pantalla teórica", pues difícilmente son implementados para efectivamente producir un patrón de distribución ideal óptimo para un caso específico. De ahí, por ejemplo, la tediosa y especulativa discusión que todavía se arrastra en los medios académicos y de planificación sobre si los rendimientos son crecientes o decrecientes en las grandes metrópolis actuales, o sobre los costos de oportunidad de invertir en zonas no desarrolladas, etc. El grado de sublimación de esta discusión llega hasta indicar que en definitiva se trata de un "conflicto entre el corto y el largo plazo". 
como método de actuar directamente sobre las variables que indican una desigualdad interregional respecto a las condiciones de vida, sobre la base de que estas acciones dejan inmodificada la situación de las variables que indican una desigualdad interregional en lo que hace a la estructura económica (composición de actividades, etc.) y que, dada la determinación (en el mecanismo hipotetizado) de las primeras por las segundas; sus beneficios se extinguen junto con los programas de inversión respectivos, mientras que para lograr efectos duraderos (y "autosostenidos") hay que trabajar sobre el segundo grupo de variables.

"Comprobado" entonces estadísticamente que un mayor grado de urbanización, una mayor densidad de caminos por kilómetro cuadrado, un mayor porcentaje de empleos industriales, una mayor productividad media, etc., están asociados a mejores indicadores de bienestar de diverso tipo, se trataría de actuar sobre los primeros para lograr que las fuerzas que producen la asociación en general impulsen, en cada región en particular, el segundo grupo de variables (este tipo de postulación es típico del denominado "desarrollismo" a nivel nacional).

Por supuesto que cabe la posición más abarcadora de considerar ambos grupos de variables como interdependientes (por ejemplo: adversas condiciones de vida en una región provocan la emigración de los mejores recursos humanos, lo que a su vez empeora las condiciones de producción, etc., etc.). Esto puede aparecer como una "dinamización" del mismo mecanismo, donde ya no se trata de una asociación sincrónica de las variables, sino de su mutuo influirse a lo largo del tiempo, lo cual, salvo ciertos desfasajes previsibles, llevaría de todos modos a producir esa asociación postulada anteriormente (sólo que sin suponer unilateralidad en la causación). En consecuencia, las propuestas implicarán trabajar sobre todo el frente de variables simultáneamente.

Si se formula entonces la existencia de un "proceso dinámico", aparecen claramente dos corrientes de pensamiento en cuanto a las tendencias que deberían esperarse si se lo deja librado a su propio accionar interno (obviamente se está hablando del mecanismo de mercado capitalista). La primera corriente apoya sus predicciones en las conclusiones de la propia teoría neoclásica (y en sus supuestos). Tal como lo plantea Williamson: "... la movilidad interna de los factores debería tender a eliminar los diferenciales interregionales de ingreso per capita, el dualismo geográfico o la polarización espacial ... la desigualdad espacial puede persistir sólo a través de retrasos en el ajuste dinámico". "De hecho, se podría apelar razonablemente al alto grado de segmentación, fragmentación y desintegración nacional general en la etapa juvenil del desarrollo nacional para predecir una creciente desigualdad durante esos primeros decenios. ${ }^{7}$

7 J. G. Williamson: "Regional Inequality and the Process of National Development: A Description of the Patterns", Economic Development and Cultural Change, Vol. 13, 1965. (Aunque algo menos mecanicista, este tipo de etapas necesarias a la Rostow también podemos encontrarlas en los primeros trabajos de John Friedmann.) 
Se fundamenta así la conocida "U" de la evolución de la desigualdad interregional, según la cual todo país pasa primero por una etapa de creciente desigualdad, luego una de estabilización y finalmente una de disminución de la misma. Como consecuencia, si se quiere acelerar el proceso lo que hay que hacer es facilitar en lo posible la libre movilidad de los factores .Esto tiene dimensiones físicas (desarrollar una malla de medios de transporte, etc.) e institucionales (desarrollar un sistema financiero de organización de las empresas y de información en general, etc.). ${ }^{8}$ Esta problemática está marcada por las concepciones neoclásicas de los procesos sociales.

Sin salir de la misma problemática, puede en cambio postularse un tipo de propuestas relativamente diferentes. Bastará con apoyarse ahora en la versión menos optimista sobre la eficiencia de los mecanismos de ajuste automático del sistema de mercado que sostienen autores como Myrdal: la causación circular acumulativa que, lejos de tender al equilibrio, alejaría cada vez más de él. Cuando específicamente estamos centrados en las desigualdades interregionales como manifestaciones de desequilibrio, el núcleo del análisis sigue siendo el de la movilidad espacial de los recursos, sólo que, ante la nueva hipótesis de tendencia, las propuestas son diversas: deben canalizarse exógenamente al mecanismo de mercado ciertos flujos de recursos hasta que se logre el equilibrio buscado y entonces el mecanismo pueda funcionar sin problemas. Es decir, deben crearse obstáculos artificiales temporarios que sin embargo respetarían las leyes de funcionamiento del mercado, de la misma manera que en el proceso de trabajo el hombre respeta y utiliza las leyes de la naturaleza. La primera causa de que un mecanismo "tan perfecto" haya dado lugar a estos problemas se encontraría en los accidentes históricos y geográficos por los cuales todo comenzó ya fuera de la posición de equilibrio (y como éste es inestable ... $)^{9}$

\section{Algunos límites de ESTA PROBlemática}

A partir de la caracterización esquemática hecha hasta aquí, podemos destacar algunas de las principales limitaciones de la problemática de las desigualdades regionales en su versión dominante.

8 Ver, por ejemplo: S. Bosier: "Industrialización, urbanización, polarización: hacia un enfoque unificado", EURE, Vol. 11, No. 5, 1972 y L. Lasuen: "On Growth Poles", Urban Studies, Vol. 6, No. 2, junio de 1969.

9 Sin duda la mejor exposición sobre esta concepción es la realizada por Carlos de Mattos: Algunas consideraciones sobre la movilidad espacial de recursos en los países latinoamericanos. Bases para su discusión, Programa Nacional de Capacitación Tecnoeconómica, México, julio de 1975. 


\section{Permanece al nivel aparencial.}

Las desigualdades entre regiones son formulados como desigualdades entre indicadores que señalan diferencias interregionales a nivel superficial (podríamos decir: "de los efectos"). Por otra parte, dichos indicadores son construidos utilizando los datos secundarios disponibles, ateniéndose por lo tanto a la percepción de los fenómenos "habilitada" por el sistema oficial de información..$^{10}$ Esto no implica que no puedan realizarse investigaciones con un sentido crítico a partir de informaciones oficiales, sino que en general las posibilidades de investigaciones con un marco teórico no apologético se ven severamente limitadas por el tipo de información disponible. Si a esto se agrega una actitud de "atenerse a los datos", los resultados son previsibles.

Lo que los métodos usualmente utilizados no llegan a descifrar es la causalidad profunda que produce estos efectos. Esto es evidente cuando se constata cuan relativa, entre estudio y estudio, es la definición de los indicadores de desigualdad y por otra parte la de las variables cuya correlación con los primeros constituiría una "explicación". El hecho de que se pase a hablar de desigualdades entre "estructuras" regionales no necesariamente supera esta limitación, pues dependerá de qué se entiende por estructura y de cómo se han delimitado las regiones.

Un ejemplo de este tratamiento superficial lo da la forma en que usualmente aparecen las determinaciones naturales en relación a la problemática de las desigualdades regionales. Mientras unas veces la existencia de determinados recursos naturales aparece como la "explicación" de la localización de ciertas actividades en algunas regiones, otras aparecen como la "decepción" del analista ante su no explotación, recurriéndose por vía de "explicación", en la mayoría de los casos, a la contraposición (por lo demás no analizada efectivamente) entre costos sociales y costos privados. En un sistema en el que no sólo el ámbito regional-subnacional sino que tampoco el nacional resultan adecuados para dar cuenta de la coyuntura y las tendencias del proceso de acumulación a escala mundial (y en particular de las estrategias de explotación de los depósitos de recursos naturales por el gran capital), basarse en la contraposición entre una falta de interés actual (real o aparente) por parte de los capitales y una "posibilidad" tecnológicamente definida por la otra, parece francamente insuficiente como vía de incorporación de los recursos naturales localizados al análisis.

10 Aún así algunos de dichos indicadores permiten denunciar con un cierto dramatismo situaciones diferenciales de la población respecto a aspectos muy claros de las condiciones de vida (por ejemplo: las tasas de mortalidad infantil), en tanto si se registran variaciones importantes entre segmentos de la población agregados territorialmente sin duda también existen entre sectores de la población en general. 


\section{El sentido clasista de las desigualdades es ocultado}

Si una de las virtudes del análisis de las desigualdades regionales es la de aproximarse al problema de las desigualdades sociales en general, dicha virtud está seriamente limitada por los supuestos en que se basa. Al tratar las unidades territoriales como conjuntos internamente homogéneos se pierde la variación intrarregional, al representar a cada región exclusivamente por el valor medio que le corresponde y analizar sólo las variaciones entre las medias regionales (véase la nota 11).

Esto no es aún más claro cuando, sobre la base de un diagnóstico de este tipo, se proponen medidas para reducir las desigualdades interregionales, que típicamente son medidas conducentes a variar más o menos directamente el numerador agregado de los diversos índices per capita. Así, si el indicador de la situación de vivienda indica que faltan más habitaciones, lanzan un plan de construcción de viviendas, cualquiera sea este, sin duda contribuirá —ceteris paribus- a mejorar el indicador.

Para un análisis típico de las desigualdades interregionales orientado por el criterio de equidad, las regiones constituyen los elementos del sistema social y son ellas las que están en posición privilegiada $o$ al final de la escala social. Consecuentemente, los conflictos asociados a estas desigualdades son "conflictos entre regiones". En otros términos, no sólo el análisis se queda al nivel superficial de una estratificación social, sino que los "sujetos" de dicha estratificación son las regiones. En tanto las regiones de una formación social constituyen aspectos secundarios de la misma ( $\sin$ siquiera entrar a discutir en qué medida la regionalización usualmente utilizada es significativa en este sentido), no hay posibilidad de descifrar los procesos sociales por los cuales se producen y perpetúan dichas desigualdades a partir de una visión del todo social como compuesto por "regiones". En lo que hace a las condiciones de vida, las desigualdades interregionales en un país capitalista son un aspecto de la estratificación social que a su vez es sólo una forma en que aparece la división en clases. Además estos enfoques no pueden entrar a un análisis en profundidad del sentido de las desigualdades interregionales en tanto comienzan negando la estratificación social misma al homogeneizar la "población" de cada región. El caso extremo del falso estatus dado a las regiones aparece cuando el análisis indica la tremenda desigualdad a que están sometidas las extensas regiones con escasa población por precisamente tener un bajo índice de densidad poblacional.

Por supuesto que en los juicios de valor y proposiciones de política puede aparecer como preocupación no ya de la desigualdad entre entes abstractos denominados regiones sino entre las poblaciones de dichas regiones. Sin embargo, estas "poblaciones", simplemente ocupan en el discurso el lugar de las regiones, sin por eso ser menos abstractas. Esto no se resuelve tampoco al manifestarse marginalmente que lo que interesa es la desigualdad entre individuos de la sociedad. 
Una población se concretiza al constituirse en un territorio determinado con sus características étnico-culturales, su historia, sus clases sociales, sus capas y fracciones y en relación a un proceso concreto de producción, circulación y consumo y a un Estado. Claro que, al partir de esta concepción, el análisis de las desigualdades debe articularse de manera muy distinta. Ya no se tratará de la diferencia entre el habitante medio de la región $A$ y el habitante medio de la región $B$, ni tampoco del grupo de individuos con tal ingreso respecto al grupo de individuos con ingreso mayor, sino fundamentalmente de las diferencias intraclases o interclases que están asociadas a determinadas localizaciones de sus miembros componentes. En qué medida y porqué determinados sectores de la clase obrera perciben un menor salario directo e indirecto al insertarse en el proceso de producción de tal o cual región, o en qué medida los terratenientes de cierta región se apropian de una menor renta que los de otra, o cuales son las condiciones diferenciales de reproducción de campesinado y su inserción en la estructura económica-social, o en qué medida y porqué las desigualdades de ingreso entre clase de una misma región son mayores que las correspondientes a otra. Este tipo de preguntas escapa generalmente a los términos de la problemática en su versión dominante. Y no puede ser para menos, en tanto su marco teórico de referencia es la teoría económica neoclásica o en todo caso la sociología funcionalista en sus versiones simplistas. ${ }^{111}$

Si otra de las virtudes del análisis de las desigualdades regionales es la de aproximarse al problema del desarrollo desigual en general, está seriamente limitado al atenerse a los términos de la concepción neoclásica de lo que es la economía. Partir de la constatación de estructuras productivas diversas, de indicadores viciados de productividad, de tasas de crecimiento diferenciales, aplicar básicamente los principios del comercio internacional óptimo al nivel de regiones intranacionales, intentar construir modelos de asignación global, sectorial y regional óptima (si nunca implementarlos de manera efectiva empíricamente), o meramente constatar la subutilización de recursos localizados, es el movimiento típico de este tipo de orientación que confunde un modelo de cálculo con su proceso social históricamente determinado.

El hecho de que se incorporen al análisis especulativo consideraciones sobre las formas actuales de la organización capitalista de la producción (monopolios, concentración y centralización, imposición de tecnologías y de pautas de consumo, la existencia de "dualismo", etc.) no necesa-

11 Tal vez valga resaltar que los límites aquí planteados no se refieren tanto al tratamiento como un todo homogéneo de cada región, que eventualmente parecería poder resolverse fragmentando territorialmente cada región o considerando estratos internos de la población en términos de intervalos de variación de cada indicador, para luego estudiar la "variación intrarregional", sino a la imposibilidad de vincular lo aparente (estratificación social en su manifestación espacial) con los procesos que lo producen (sociedad de clases), la que es exacervada cuando las regiones se toman como sujetos sociales. 
riamente modifica los términos fundamentales de la problemática, en tanto la explicación de las desigualdades interregionales sigue limitada básicamente al funcionamiento de un modelo económico ahistórico que si bien es más "realista", evita entrar en las cuestiones esenciales de una sociedad capitalista.

Lo "histórico" aparece en algunos trabajos como una secuencia más - menos estereotipada de etapas por las cuales habría pasado la economía nacional (agrario-exportador, sustitución de importaciones, etc.) y en el intento de establecer relaciones entre estas etapas y la situación de desigualdad regional interna. Incluso es posible encontrar una mayor riqueza y concreción del análisis para el periodo colonial o para el siglo pasado, pues al llegar a las etapas más recientes se comienza a recurrir a pseudo explicaciones tales como la del proceso de causación circular acumulativa, a las llamadas economías externas, y a otros aspectos particulares del mecanismo económico que son llevados al estatus de gran hipótesis explicativa (nunca corroborada por otra parte), del fenómeno de las desigualdades regionales.

\section{La ausencia de "lo político" y la concepción del Estado}

Una caricatura del método que estamos caracterizando lo representaría como descubriendo desigualdades interregionales que se atribuyen en el mejor de los casos al funcionamiento de un mecanismo económico operando en una suerte de vacío político-social. Al momento de las propuestas aparece el "Estado", como superelemento de la sociedad con cierta capacidad autónoma de maniobra. El analista (en la versión intervencionista) supone que el Estado debería asumir una función objetivo centrada en criterios de equidad o de crecimiento económico y aconseja acciones (usualmente programas de gasto público, pero también variaciones paramétricas reguladoras del sector privado, como las propias de la política bancaria, fiscal, y regulaciones legales de diversos tipo) que conducirían a obtener los objetivos buscados. Marginalmente, aparece algo denominado "político", en tanto se considera que las poblaciones de las regiones desfavorecidas podrían presionar (a través de sus representantes) sobre el Gobierno central para obtener un trato más favorable. Ni "lo político" ni "la política" forman parte del análisis en la versión bajo consideración.

El hecho de que junto a este tipo de análisis se denuncie el sentido promonopolista o a favor de tal o cual fracción de la burguesía de las intervenciones del Estado, no garantiza una superación de esta problemática en cuanto persista una visión instrumentalista del Estado. Es más, en el modelo de base puede llegar a "incorporarse" al Sector Público como parte orgánica del aparato económico de la sociedad, y mostrar como realimenta la generación de desigualdades regionales, sin por eso superar esta limitación de la problemática. 


\section{LAS “TENSIONES" INTERNAS DE LA PROBLEMÁTICA}

Lo que antecede es una esquemática caracterización de los términos de la problemática tal como puede ser extraída de los resultados de la práctica misma de un sector importante de quienes han prestado atención al problema de las desigualdades regionales. Sin embárgo, como anticipáramos, no pretendemos que coincide punto por puntojcon un método estandar aplicado recurrentemente, ni que es propugnada tal cual como "el método" por ningún autor particular.

Propugnamos sí, que lo expuesto refleja ajustadamente características fundamentales de la problemática en su versión dominante. Sin embargo, en tanto se trata de un modelo ideal, las prácticas técnicas que pueden ser caracterizadas como reguladas por esta problemática admiten cierta variación (tal como se fue marcando en el acápite anterior) sin por eso superar los límites de la misma. Aún así, tales desarrollos son importantes, pues constituyen un paso para dicha superación. En particular, una aplicación seria de estos métodos debería llevar, por sus propias contradicciones internas, a generar "tensiones" que impulsen a superar sus límites (o, en el peor de los casos, a explicitarlos). Para ésto bastaría, por ejemplo, con llevar hasta sus últimas consecuencias cualquiera de los criterios básicos esbozados más arriba: el de equidad o el de eficiencia en términos de crecimiento.

Esto ocurre si en lugar de seguir aplicando mecánicamente tal o cual fórmula para constatar la existencia de desigualdades regionales en las condiciones medias de vida, y luego establecer como objetivo un cambio en los indicadores medios y proponer algunas acciones que si alguien las realizara tenderían a tal cambio, el analista se repregunta por el objetivo superior que supuestamente regula el análisis y su efectiva relación con la propuesta realizada: ¿Qué significa efectivamente la desigualdad social y hasta dónde puede eliminarse en una sociedad capitalista? Esto nos lleva a formular la relación entre las diversas formas de la estratificación social y las relaciones de producción. En cuanto la visión atomística de la sociedad es sustituída por una concepción que al menos tenga en cuenta la existencia de clases sociales, de fracciones y capas dentro de las mismas y de sus relaciones con los aparatos de Estado, el método arriba esbozado comienza a traslucir un carácter ideológico. Las desigualdades interregionales dejan de ser un problema provocado por ciertas fricciones o desfasajes en la dinámica del mecanismo económico para ser vistas como características estructurales en una sociedad dominada por las relaciones capitalistas de producción. Las políticas del Estado dejan de ser consideradas como perfectibles con más y mejor información, y se vislumbra la funcionalidad compleja del discurso de la planificación. La visión de las regiones como sujetos comienza a perder sentido.

Asimismo, si en lugar de seguir considerando los criterios de eficiencia desde la perspectiva de un modelo de asignación óptima de recursos se 
pasa a examinar el proceso mismo de desarrollo de la capacidad de producción de un país, y los obstáculos que el sistema social le impone, ocurre otro tanto. Por una u otra vía, aparece en escena la delicada cuestión del modo de producción capitalista...

Una vez conscientes de los límites de la problemática en su versión dominante (es decir: de "lo que no puede ver") pueden adoptarse diversas posiciones. ${ }^{12}$ Una alternativa es simplemente decir: si las desigualdades regionales se producen en el seno de un sistema capitalista que por su misma naturaleza pone obstáculos a la superación de las mismas, entonces la única manera de resolver el problema es cambiar el sistema y todo lo que se haga dentro del mismo será inútil. Obviamente ésta no es una propuesta de acción al sector público y remite exclusivamente a otro tipo de práctica supuestamente pura: la política. Por otra parte, para ser consecuente con esta posición, hay que arrojar al bebé con el agua y abandonar el análisis de las desigualdades regionales.

Otra alternativa es adoptar la posición de "separar", en una coexistencia pacífica, la práctica política de la que hasta ahora nos venía ocupando. En tanto somos claramente conscientes de los límites del sistema social, y mientras este no cambie, nos movemos según sus reglas. Tal como lo señala de Mattos: “. . . debe tenerse en cuenta que la libre movilidad interregional de recursos está condicionada y acotada por las relaciones de producción inherentes a una economía de mercado. Siendo ello así, las medidas del programa de política económica del plan regional podrán buscar la modificación de las mencionadas condiciones y mecanismos dentro de los márgenes viables a dicho tipo de economía. Ello implica que las medidas adoptadas no pueden apuntar a la alteración, más allá de ciertos límites, de los elementos definitorios del sistema económico en cuyo seno se está trabajando, desde que partimos del supuesto que el cambio de sistema socioeconómico sólo es materia de decisión en un plano estrictamente político. En síntesis, los instrumentos que se articulan en el programa de política económica deben ser concebidos y ubicados en el área de factibilidad propia a una economía de mercado $\mathrm{y}$, por consiguiente, no puede afectar la esencia del funcionamiento de una economía de este tipo" [op. cit.].

Ambas posiciones tienen una característica común: la de suponer que las prácticas sociales pueden separarse en tipos puros: la práctica política, que tiene como campo específico las cuestiones relativas al poder; las prácticas "técnicas", que por su carácter neutral no pueden ni deben tener efectos políticos. En consecuencia el Estado es un bastión que a lo sumo puede ser asaltado desde "afuera" y estar "dentro" implica necesariamente complicidad o en el mejor de los casos neutralidad.

12 Sin duda que a esta concientización contribuye no sólo la insatisfacción intelectual con los modelos teóricos de base, sino la constatación de que las propuestas de política suelen quedar en las bibliotecas y que incluso las implementadas no tienen los resultados esperados. 
Esta es la disyuntiva que se suele proponer a quienes, ubicados dentro de alguno de los aparatos de Estado (los organismos de planificación, las universidades) han intentado dar cuenta de las desigualdades regionales y están ya sobre los límites de la problemática tradicional.

\section{LA POSIBILIDAD DE REDEFINIR LA PROBLEMÁticA ${ }^{13}$}

Si admitimos que no hay prácticas "puras", sino que aún una práctica fundamentalmente técnica tiene dimensiones políticas e ideológicas, y que las prácticas predominantemente políticas o ideológicas no se efectúan en un vacío institucional, o "fuera del sistema", sino que es desde su mismo interior contradictorio que se va gestando el cambio, la disyuntiva anterior nos aparece como falsa. No se trata entonces de optar entre analizar, explicar y contribuir a pensar como morigerar las desigualdades interregionales teniendo cuidado de no traspasar los límites políticos e ideológicos del sistema imperante o, en cambio, "dedicarse a la política" (o hacer ambas cosas con distintos horarios), sino de advertir que las desigualdades interregionales constituyen contradicciones secundarias de un sistema social atravesado justamente por una red de contradicciones articuladas. Si nos preocupan las desigualdades regionales, y el análisis nos lleva a la conclusión de que el mismo sistema social las realimenta de modo que difícilmente puede esperarse un cambio importante en un lapso políticamente significativo, la cuestión es encontrar el lugar que estas contradicciones ocupan en el conjunto de contradicciones articuladas y, en particular, qué relación tienen con la contradicción fundamental que hace al sistema de poder mismo.

En otros términos, consideramos que aún desde una teoría que no sea la del statu quo puede tener relevancia considerar el problema de las desigualdades interregionales, por supuesto que ahora en términos de una problemática distinta.

Aparentemente, una buena vía para comenzar a reformular la problemática sería, a partir de la constatación de la existencia de desigualdades regionales, preguntarse por qué mecanismos el capitalismo produce desigualdades, por supuesto que a partir de una teoría no apologética de su funcionamiento. En otros términos, fundamentar la necesidad de las desigualdades interregionales en formaciones sociales capitalistas.

Este análisis sería realizado fundamentalmente al nivel de modo de producción. Pasando ya al nivel de formación económico social, la cuestión de la coexistencia de diversos modos de producción, ya sea vista en

\footnotetext{
13 Lo que sigue se refiere a los países capitalistas de Latinoamérica. Para el caso de Cuba obviamente la problemática debería ser encarnada de manera totalmente distinta. Un trabajo interesante sobre el tema es el de David Barkin: "Confronting the Separation of Town and Country in Cuba", en L. Sawers y W. Tabb (Comps.), Conference on Urban Political Economy, Oxford University Press, 1977.
} 
términos de una incompleta difusión del capitalismo o de la funcionalidad de formas atrasadas de producción para el mismo proceso de acumulación capitalista, pasarían a vincularse con la cuestión de las desigualdades regionales en particular. Sobre esta base se procuraría explicar las diferencias interregionales específicas en una sociedad concreta, incorporando otras determinaciones relevantes. Tarde o temprano aparecerá la pregunta acerca de qué ocurre al respecto en otro tipo de formaciones sociales existentes, o en otras fases de capitalismo, etc., etc. Aunque éste parecería el camino más seguro para avanzar, a nuestro juicio el planteamiento de la cuestión regional en América Latina requiere otro tipo de aproximación (conjugadamente con el anterior) centrado en las determinaciones políticas.

En primer lugar, la cuestión no es preguntarse por qué en el capitalismo se producen desigualdades entre regiones sino por qué produce $d e-$ terminadas desigualdades entre regiones. $\mathrm{Y}$ no nos referimos aquí solamente a la magnitud o al peculiar patrón espacial de dichas desigualdades, sino fundamentalmente a su contenido social especifico. Preguntarse por las desigualdades regionales en general fácilmente nos llevaría al callejón sin salida de concluir que la desigualdad espacial es un fenómeno de carácter universal. En segundo lugar, la cuestión no es preguntarse cómo puede hacerse desde el Estado para paliar las desigualdades interregionales, sino por qué en determinada coyuntura la existencia de ciertas desigualdades se conistituye en un "problema", a nivel del Estado (en lo que hace a la política económica la más de las veces, pero también, otras, en directa vinculación con el aparato de represión).

En resumidas cuentas nos preguntamos cómo en determinada coyuntura las contradicciones entre intereses de diversas fracciones de la burguesía, entre si y con el campesinado, el proletariado o alguna de sus capas especificas, aparecen como contradicciones interregionales, y bajo qué condiciones llegan a constituir una cuestión que exige regularse a nivel del Estado.

Estamos acostumbrados a oir hablar del fracaso de las políticas destinadas a disminuir las desigualdades regionales aun cuando este objetivo haya sido incorporado al folklore de la planificación. Muchas veces se afirma que en realidad no hay tal fracaso, pues el carácter de la planificación en nuestros países no es el de efectivamente "racionalizar" ciertos aspectos de la estructura económica, ni mucho menos regular las contradicciones existentes, sino que es un mero discurso ideológico. En consecuencia , podría concluirse que la cuestión de las desigualdades regionales ha aparecido a nivel de los aparatos del Estado por un puro desarrollo relativamente autónomo de la ideología de la planificación, impulsado desde medios académicos universitarios y desde la misma burocracia embarcada en dichas tareas.

Si reflexionamos sobre el sentido de la planificación en nuestros países, en muchos casos podríamos fácilmente aceptar esta caracterización, que 
establecería una neta diferencia con la caracterización que autores como Castells hacen de la planificación en Francia como una parte del proceso político ${ }^{\mathrm{H4}}$ Sin embargo, cabría hacer un par de aclaraciones: esta caracterización puede ser válida cuando partimos de una visión de la planificación como proceso globalizante, pero, sin embargo, no aplicarse a determinadas instancias de planificación en campos muy específicos (sectoriales, por ejemplo). Por otra parte, tal vez una característica aún perseverante en muchos de nuestros países es que por un lado corre lo que se llama planificación, en manos del sector de la burocracia denominado consecuentemente "los planificadores" y por otro van las políticas del Estado, en muchos casos sin articulación eficaz entre ambas. Esto parece particularmente válido para el caso de la denominada planificación regional o territorial (aunque haya importantes diferencias entre distintos países y épocas). Ahora que en lo que hace a las políticas del Estado, ya no es tan fácil aceptar que no forman parte del proceso político. En otros términos, podría pensarse que hay un desfasaje entre prácticas de planificación con un débil componente político y un mayor peso ideológico, y las prácticas directamente ligadas a la gestión de las políticas con una necesaria tendencia a que ambas converjan en los términos de Castells. Desde este punto de vista, parecería que preguntarnos por el momento preciso en que las desigualdades regionales aparecieron en escena como "problema" nos daría una falsa respuesta, por su carácter aún predominantemente ideológico, en cuanto estamos ubicándolo en una fase de planteamiento y denuncia del problema (aunque sea dentro de los mismos aparatos del Estado), sin consecuencias efectivas sobre las políticas del Estado (es decir: sin que efectivamente sea considerado como un "problema").

Sin embargo, creemos que un análisis histórico de la cuestión, no ya de los discursos formalizados bajo el título de desigualdades regionales, sino de coyunturas en las cuales efectivamente se han presentado conflictos de intereses con una clara expresión regional que han requerido de una intervención específica del Estado, nos permitiría ver que, con altibajos, el "problema" ha estado presente, aunque sin necesariamente organizarse como una política global expresamente tendiente a regular las contradicciones expresadas bajo la forma de desigualdades regionales. En adición, un análisis histórico nos permitiría percibir las diversas articulaciones que pueden darse con el sistema de contradicciones.

Por ejemplo, cómo podríamos dejar de distinguir situaciones como las siguientes:

Situación $A$ : Ante la crisis por la que pasa la industria localizada en cierta región periférica, los empresarios afectados presionan para que se derogue una reglamentación zonal por la cual sus obreros perciban un salario mayor. Con el apoyo de algunos sectores de la pequeña burguesía, el movimiento obrero de la región comienza un proceso de movilización,

14 Ver Castells y Godard: Monopolville. L'Entreprise, l'Etat, l'Urbain, Mouton, París, 1974. 
con paros y concentraciones populares, defendiendo la reglamentación y demandando del gobierno central medidas especiales del apoyo a la industria en lugar de una baja de sus salarios. En tanto las movilizaciones van en aumento y se van incorporando otros sectores y nuevas reivindicaciones, el gobierno comienza a reprimir, siendo por momentos desbordado por la movilización masiva de la población.

Situación $B$ : Llegado el momento de la cosecha de la producción frutícola de una región periférica ${ }_{y^{\prime}}$ el sector más concentrado del empaque y la comercialización presenta una posición de fuerza ante el gobierno nacional en su reclamo de una disminución de los substanciales impuestos a las exportaciones. Se organiza una movilización que incluye a transportistas, pequeños productores, trabajadores agrícolas vinculados directamente a la actividad y al que se suman los comerciantes de la región. Se ocupan caminos regionales, se toman edificios y se paraliza la cosecha hasta que el gobierno acepta negociar.

Situación $C$ : En una región periférica reconocida como región "problema" por la extremada presión demográfica sobre la tierra e indigencia de amplios sectores del campesinado, un grupo importante de campesinos sin tierra ocupan por la fuerza una zona de gran productividad dedicada a cultivar productos de exportación, amparándose en los principios de justicia social declarados por el gobierno y en expresas disposiciones de la ley de reforma agraria. Esto provoca la reacción de la oligarquía agraria que poseía esas tierras y se extiende inmediatamente al grueso de la burguesía que llega a organizar ceses de actividad a nivel nacional para presionar al gobierno, que finalmente dispone que se vuelva a la situación anterior, pasando el asunto a la instancia judicial. La movilización campesina recibe declaraciones de apoyo de diversas organizaciones pero sin acciones de fuerza efectivas.

Situación D: El General a cargo de la represión militar de la guerrilla en una región agraria de monocultivo, empobrecida a raíz de medidas de política económica racionalizadora de la producción del sector, hace un severo llamamiento a la burguesía con intereses locales para que colabore para lograr el desarrollo regional so pena de que los esfuerzos para terminar con la guerrilla sean inútiles.

Situación $E$ : El gobierno nacional pone en marcha un plan masivo de inversión e infraestructura en una región periférica con escasa población y abundantes recursos minerales de interés estratégico para las empresas internacionales que lo requieren para continuar con su proceso de acumulación a escala mundial. El proyecto es presentado como conducente al objetivo de acabar con las desigualdades regionales entre la zona central y la periferia, y cuenta con el apoyo de las corporaciones empresarias nacionales en tanto el Gobierno se limite a hacerse cargo de los sectores no rentables del proyecto. Los sectores progresistas del aparato político ven anulada su capacidad de control del proyecto a partir de una disposición 
jurídica que da plena autonomía a la Corporación Regional especialmente creada para administrar el proyecto.

Este tipo de situaciones, presentadas aquí a nivel "periodístico", constituyen materia prima para el análisis (enmarcado en un análisis de la coyuntura general) de la cuestión de las contradicciones sociales complicadas con desigualdades regionales que está aún por hacerse de manera sistemática. Sin esta base, y mientras sigamos concentrándonos en análisis de variaciones interregionales de indicadores, por atípicos que estos indicadores puedan ser, será difícil superar una situación de inconformidad con los términos de la problemática dominante pero sin posibilidad de superarla no especulativamente.

En particular, para investigar la relación de las desigualdades regionales con las políticas del Estado, no pueden meramente superponerse -a los resultados de un análisis de la relación entre proceso de acumulación capitalista y organización espacial- una concepción general del Estado y la planificación, e intentar especificarla para el caso particular de estos "problemas". Se sugiere que habría que empezar por señalar, a través de un análisis histórico (obviamente regulado desde un marco categorial y conceptual), en qué situaciones particulares las contradicciones de diverso orden que atraviesan nuestras formaciones sociales han aparecido como contradicciones interregionales y bajo qué condiciones coyunturales han llegado a constituirse en "problemas" que requieren la expresa regulación a nivel de Estado.

\section{La Cuestión Regional en AmÉrica Latina}

Si nos atenemos a los juicios críticos esbozados en los acápites anteriores, la problemática regional en América Latina estaría aún por ser formulada. Pero esto no debería ser hecho sobre la base de especulaciones sino con base en investigaciones específicas que en su paulatina articulación fueran dando forma a los términos de la Cuestión Regional en América Latina. Tal es el sentido de este proyecto de trabajo cooperativo entre estudiosos de latinoamérica que han estado abocados frontal o tangencialmente a estos temas, ya sea un país o zona particular. No se trata de "liquidar" el tema, de cerrar la problemática, sino de abrirla, de modo que a nuestro mejor saber y entender podamos señalar las vías y métodos prioritarios para futuras investigaciones en el campo.

Como siempre ocurre, aun cuando se desconozca el resultado al que una investigación ha de conducir, su misma puesta en marcha está ya orientada por ciertos presupuestos. En este caso, y dado que se trata de un esfuerzo colectivo entre casi cuarenta personas, muchas de las cuales tal vez no se conozcan, la coordinación del proyecto ha debido dar un primer paso en sentar tales presupuestos, y esto se refleja de hecho en la 
organización misma de los temas a los que se llegue en 1978.* Muy probablemente sea difícil reconocer en el resultado la intención inicial, pero justamente de eso se trata, de poner en movimiento mancomunado energías de investigación que permitan efectivamente establecer un punto de partida no especulativo para futuras investigaciones y acciones.

Al organizar la temática ha sido nuestra intención que fueran traídos a la discusión de marzo 1978 los principales elementos constitutivos a nuestro juicio de la cuestión regional.

En primer lugar, si en la base de esta problemática está la relación entre procesos sociales y organización espacial, qué más apropiado que comenzar a través de investigaciones históricas, que nos permitan captar la variación en dicha relación a lo largo de la historia latinoamericana. La segunda parte del proyecto apunta justamente a traer esa relación a la mesa de discusión. ¿En qué difiere la lógica de la organización espacial en las sociedades indígenas precolombinas, luego en el régimen colonial, español o portugués y posteriormente bajo el imperialismo en sus distintas etapas? ¿en que medida las formas espaciales preexistentes son destruídas, asimiladas, desplazadas por las nuevas formas sociales en desarrollo?, ¿en qué sentido determinan desarrollos futuros?, ¿qué variaciones (no precisamente lineales) ha tenido la cuestión regional durante el proceso de creación, consolidación y posterior desarrollo de los estados nacionales latinoamericanos?, son algunos de los aspectos de la cuestión a discutir.

En la tercera parte del proyecto se intentaría incorporar algunas de las principales determinaciones que hacen a la Cuestión Regional en Latinoamérica como un todo, en lo posible registrando las variaciones entre subregiones o países. El estado actual de las investigaciones forzará muy posiblemente a tener que combinar proposiciones que intenten referirse a América Latina en su conjunto con cierta fundamentación empírica fragmentada o muy concentrada en el estudio de un número pequeño de casos. Justamente se trata de crear las condiciones para una futura superación de esta situación, formulando las preguntas adecuadas y avanzando hipótesis cuando se pueda.

Aquí sería deseable mantener a la vista el complejo determinismo que hay en la cuestión, pero centrando cada contribución en un aspecto principal. Así, las determinaciones estructurales que hacen a una posición subordinada en el sistema capitalista mundial en su etapa actual y sus tendencias previsibles en lo que hace a la organización espacial, se articularían con la cuestión de la disponibilidad de recursos naturales en el continente, con la existencia de grandes comunidades indígenas en determinadas regiones, y con una dinámica demográfica que -con diferencias entre países- es una característica importante del proceso actual.

* N. de E. Este trabajo fue realizado durante 1977. Se hace aquí referencia al Seminario sobre la Cuestión Regional en América Latina, que tuvo lugar en El Colegio de México en abril de 1978 y cuyas conclusiones generales serán publicadas en el siguiente número de la revista. 
En la cuarta parte se intenta dar a la cuestión agraria en América Latina la importancia que tiene en relación a la problemática regional; aspecto éste relativamente relegado o no articulado apropiadamente en las corrientes predominantes en los países centrales donde lo urbano ha ocupado el centro de la escena. Nuevamente la forma de articulación de ambas cuestiones no es uniforme y registra variaciones importantes entre países, que sería necesario destacar.

La quinta parte, al conformar un núcleo temático con las determinaciones políticas, responde por un lado a la convicción de que es necesario superar un cierto economicismo, imperante en buena parte de los estudios sobre problemas regionales $\mathrm{y}$, por otro, a la necesidad de investigar la eficacia relativa de diversas vías de acción social destinadas a acelerar o consolidar determinadas tendencias del desarrollo social, con especial referencia a los movimientos sociales orientados hacia aspectos estrechamente vinculados con la problemática regional.

La sexta parte esta destinada principalmente al análisis de los efectos ideológicos que se producen al formularse los "problemas regionales" tanto a nivel teórico como a nivel de las políticas del Estado. Este análisis se basaría en un examen de lo que ha constituído efectivamente la práctica de la planificación regional en los países de América Latina, el tipo de teorías que la acompañan así como un análisis del contenido de los programas de formación especializada para funcionarios públicos, fundamentalmente en cursos de organismos internacionales, por donde han pasado buena parte de los especialistas actualmente en los aparatos de Estado latinoamericanos.

Por último, en la séptima parte se presentarán los términos específicos de la cuestión regional en cada país latinoamericano. Aquí es donde se nos presentaría una oportunidad de destacar las formas particulares de articulación de las complejas determinaciones que hacen a la cuestión regional, con base en el estudio de sociedades concretas, y sobre esta base recomponer críticamente las proposiciones generales que puedan haberse generado en los otros estudios. 